\title{
Constructing value signals for food rewards: determinants and the integration
}

Shinsuke Suzuki ${ }^{1}$

${ }^{1}$ Brain, Mind and Markets Laboratory, Department of Finance, Faculty of Business and Economics, The University of Melbourne, Parkville, VIC 3052, Australia.

Correspondence to: Shinsuke Suzuki (shinsuke.szk@gmail.com)

Highlights:

- Subjective values of foods guide our dietary choice.

- The food value is constructed by integrating its constitutive attributes.

- The attributes include visual, nutritive, and abstract features of the food.

- The integration is implemented in a hierarchically-organised brain network.

Conflict of interest statement: No conflicts declared.

Acknowledgments: This work was supported by the Mishima Kaiun Memorial Foundation.

\begin{abstract}
Based on recent findings in human decision sciences, we propose a neurocomputational mechanism for the construction of value signal of a food reward. In dietary choice, an individual computes the subjective value of a food by integrating its nutritive (e.g. carbohydrate) and abstract features (e.g. healthiness) inferred from the low-level visual information (e.g. colour). This process is implemented in a hierarchically organised brain structure, including early visual, parietal, and prefrontal cortices. We also discuss the effects of other factors such as cognitive controls and reinforcement learning. The proposed architecture for food valuation would provide a stimulating hypothesis for future studies in diverse research fields.
\end{abstract}




\section{Introduction}

Food choices ('what to eat') are among the most fundamental decisions people make on a daily basis [1]. Such decisions are thought to be guided by subjective preference, called value or utility [2]. For instance, an individual selects food items during grocery shopping based on the computed value signals [3]. Maladaptive food valuation is often associated with the development of obesity and eating disorders [4,5]. Given the importance of dietary habits in our health, the valuation of food is a central issue in decision sciences, including neuroscience, psychology, and psychiatry [6-8].

Human neuroimaging research to date has discovered that $\mathrm{WHICH}$ brain regions represent subjective values of food [9] by employing functional MRI combined with wellestablished behavioural experiment paradigms (e.g. Becker-DeGroot-Marschak auction [10]). In particular, the ventromedial prefrontal cortex (vmPFC), including the adjacent medial orbitofrontal cortex (OFC), encodes value signals for food rewards as well as for other types of rewards such as money, trinkets, and leisure activities [11-13]. However, we know little about HOW the human brain computes food values (Figure 1) [14]. Accumulating evidence in decision neuroscience, although indirect, suggests that food values could be constructed by integrating multiple attributes such as perceived tastiness, healthiness, macronutrients, and visual appearance $[3,14,15]$. Here, we review recent findings on food valuation in the human brain and discuss a possible mechanism of food value computation.

\section{Perceived tastiness and healthiness}

Seminal works have suggested that one integrates information about the tastiness and healthiness to compute the overall value of the food [16-21]. On comparing the effects of the two attributes, Sullivan et al. found healthiness contributes to food choice to a smaller extent and longer latency as compared to that of tastiness [22]. Furthermore, a recent study provided a mechanistic account of the behavioural pattern using the multi-attribute timedependent drift-diffusion model [23]. The tastiness and the healthiness are encoded in the vmPFC and the lateral prefrontal cortex (PFC) respectively $[16,18]$ at the time of dietary choice, while the overall value signal is represented in the vmPFC [16-18]. Moreover, the behavioural and neural effects of healthiness on dietary choice were found to be modulated by cognitive regulations (e.g. attention toward healthiness) $[16,18]$ as well as individual differences in the preference for healthy foods [17] and the Body-Mass-Index [24]. Xue and colleagues [25] compared the multi-variate neural representations of the tastiness and the healthiness 
between women with anorexia nervosa (AN) and the healthy controls. They showed that the representation of healthiness in the OFC more accounted for food preference among patients with AN than healthy individuals, suggesting a role of over-consideration of the healthiness in maladaptive food choice in AN (see also [26]).

It is worth noting here that perceived tastiness and healthiness are high-level abstract features of food that could be attributed to lower-level features such as macronutrients, flavours, and visual appearance. However, the mechanism involved in the establishment of these high-level beliefs from lower-level features in the brain remain unclear.

\section{Anticipated nutritive contents}

In general, people tend to prefer high-calorie foods (i.e. 'eat for calories'). Food preference has, at least in part, evolved for efficient intake of the necessary nutrients for survival $[27,28]$. Based on this concept, Tang et al. [29] demonstrated the correlation between subjective value for a food item (i.e. willingness to pay) and caloric density, even after controlling for the effect of the retail price. Furthermore, the caloric information as well as the subjective value were found to be encoded in the vmPFC.

Suzuki et al. [27] examined the computation of food values based on nutritive attributes. They found that the subjective value of a food item can be predicted by a linear combination of the nutrient factors anticipated from the visual appearance (note: people are capable of inferring the nutrient information from a picture of the food rapidly [30]). Moreover, multi-voxel pattern analyses on the neuroimaging data revealed that the subjective value was encoded in both the lateral and medial parts of the OFC; however, the nutrient information was encoded only in the lateral OFC. The functional dissociation between the lateral and medial OFC is consistent with the anatomical structure [31]: the lateral part receives extensive inputs from multiple sensory areas (e.g. gustatory, olfactory, and somatosensory cortices), while the medial part sends outputs to the hypothalamus and brain stem. The dissociable roles are also consistent with another line of research [32] demonstrating identity-specific value coding in the lateral OFC (e.g. the value of a specific flavour) and the identity-general value coding in the medial OFC.

To further explore the functions of OFC and other brain regions in food valuation, this study [27] also tested the representation of low-level visual features of the food item (e.g. luminance and local contrast) in the OFC as well as other regions, including the primary visual 
cortex (V1) and the posterior parietal cortex (PPC). The additional analysis revealed that the lateral OFC encodes nutrient information but not low-level visual information. On the other hand, V1 predominantly encodes visual information, while PPC encodes both. These findings together would suggest a hierarchical architecture for food valuation: the low-level visual information of a food item represented in the $\mathrm{V} 1$ is converted into the nutritive attributes in the PPC and stored in the lateral OFC, which is further transferred to and integrated in the medial OFC (vmPFC) to compute the overall value signal (Figure 2a).

Using a similar experimental paradigm, another study [33] demonstrated the supraadditive interaction effects of fats and carbohydrates. Participants in the experiment preferred foods containing both fats and carbohydrates over equally caloric foods containing either fat or carbohydrate alone, which was in turn reflected by an elevated activation of the brain reward circuit (e.g. dorsal striatum). This result provides a potential explanation for the excess consumption of high-fat/high-carbohydrate food products (e.g. potato chips).

\section{Hierarchical construction of value signals}

Upon implementation of hierarchical architecture (Figure 2a) for food valuation, (i) low-level visual information of a food item would predict its nutrient information and subjective value, and (ii) the value prediction from the visual information would be less accurate than that obtained from the nutrient information. We examined the reasoning by re-analysing the behavioural data in a previously-published study [27]. Linear regression demonstrated that low-level visual information (colour intensities, hue, saturation, brightness, and local contrast) significantly predicted the nutrient information as well as the subjective value (Figure $2 b$ ). Furthermore, the accuracy of the value prediction from the visual information was lower than that of the predicted value obtained from the nutrient information (Figure $2 b$ ). These results are consistent with hierarchical information processing for food valuation.

Remarkably, the hierarchical architecture (Figure 2a) resembles the proposed mechanism for the valuation of the visual arts. Recent studies $[34,35]$ have suggested that hierarchical information processing underlies the valuation of visual arts by correlating a deep convolutional neural network (DCNN) model with behavioural and neuroimaging data. DCNN is a machine learning algorithm that performs visual object recognition tasks, as accurate as humans [36]. Notably, the algorithm hierarchically processes visual information from the early layers to deeper layers in the neural network. Therefore, DCNN would be relevant for a 
computational model of hierarchical information processing in the biological brain [37]. ligaya et al. $[35,38]$ demonstrated that DCNN can predict subjective values of arts with the earlier layers containing information on low-level visual features (e.g. mean hue and mean saturation) and the deeper layers containing information on high-level features (e.g., 'abstract or concrete' and 'dynamic or still'). Furthermore, they found a significant correspondence between the earlier (deeper) layers in the artificial neural network and visual cortical (parietal-frontal) regions in the human brain. The similarity between the valuations of food items (Figure 2) and visual arts $[34,35]$ implies that the hierarchical processing of information is a ubiquitous structure for constructing value signals independent of the type of object [14]. A fruitful avenue for future research would be to elucidate the hierarchical mechanism for food value computations by leveraging DCNN models.

This review has thus far mainly focused on 'bottom-up (feedforward)' food valuation based on visual sensory input. However, food valuation would be affected by other factors such as choice-induced preference changes [39] and associations of the food item with a memory of past positive/negative experiences [40] as well as social influences [41]. For example, Barron et al. [42] suggest that the value of a novel food that has not been previously tasted can be inferred by retrieving relevant memories in the past from the hippocampus and mPFC. These effects may work as feedback modulations in the valuation system (e.g. recurrent neural networks).

\section{Update of the value through consumption/tasting experience}

Another line of literature in neuroscience has investigated the value signals evoked by actual consumption/tasting of food. Oral sensory representation of fat and sucrose was found to be represented in the vmPFC [43], while the pleasantness of the oral fat texture is represented in the vmPFC and the lateral OFC, as well as their functional connectivity with the oral somatosensory cortex $[44,45]$. Another study showed that the lateral OFC encodes the pleasantness of tastes, while the intensity of taste is encoded in the insular cortex and amygdala [46].

Recently, two research groups have independently identified the primary gustatory cortex in humans $[47,48]$. Utilising ultra-high-resolution $\mathrm{fMRI}$ combined with multivariate analysis, Chikazoe et al. [47] demonstrated that taste qualities (sweet, salty, bitter, and sour) are represented in the insula, which has been previously implicated in the primary gustatory 
cortex in non-human animals. A subsequent study [49] showed that the gustatory cortex, insula, responded both to viewing images of foods and to tasting the foods, but in distinct patterns of neural activity (see also [50]).

Neural responses to consuming/tasting are thought to be more related to the postdecisional evaluation of food rewards (rather than food choices). The reinforcement learning account of animal conditioning posits that the value of a food is updated by the discrepancy between the value of the actual reward consumed and the value estimated (called 'reward prediction error') [51]. One interesting question is whether value learning requires oral sensing of taste/flavour [6]. One possibility is that oral sensing leads to hedonic experiences and thus drives subsequent behaviours as a reinforcer. An alternative is that calorie intake (i.e. nutritional value) is sufficient for regulating future behaviour, and oral sensing is unnecessary. Consistent with the latter possibility, animal research consistently demonstrated that intragastric infusion of nutrients (e.g. glucose) without oral sensing can form conditioned behaviours [6,52], that is, animals develop preferences for the choice options paired with the infusion of nutrients directly into their stomach. These findings support the possibility that postoral signals, but not necessarily oral signals, drive value updating, and shape future behaviour.

\section{Conclusion}

In this study, we have carefully reviewed recent findings from human neuroimaging studies on food valuation. In particular, the review focuses on how multiple attributes are integrated to construct the value signal from the visual sensory input. We propose a potential mechanism: i.e. hierarchical construction of value signals. To summarise, low-level visual features are converted to the anticipated nutrient information and high-level abstract features, such as tastiness and healthiness, and then integrated to compute the overall value. The bottom-up (feedforward) information processing is implemented in a hierarchically organised brain network from the visual cortices, the parietal cortex, and the lateral PFC/OFC to the vmPFC. Further studies are required to validate the proposed mechanism and elucidate the effects of other factors, such as cognitive controls, choice-induced preference change, and reinforcement learning on the valuation process. 


\section{References}

1. Wansink B, Sobal J: Mindless Eating. Environ Behav 2007, 39:106-123.

2. Ballesta S, Shi W, Conen KE, Padoa-Schioppa C: Values encoded in orbitofrontal cortex are causally related to economic choices. Nature 2020, 588:450-453.

3. Motoki K, Suzuki S: Extrinsic Factors Underlying Food Valuation in the Human Brain. Front Behav Neurosci 2020, 14:131.

4. Foerde K, Walsh BT, Dalack M, Daw N, Shohamy D, Steinglass JE: Changes in brain and behavior during food-based decision-making following treatment of anorexia nervosa. $J$ Eat Disord 2021, 9:48.

5. Uniacke B, Slattery R, Walsh BT, Shohamy D, Foerde K, Steinglass J: A comparison of food-based decision-making between restricting and binge-eating/purging subtypes of anorexia nervosa. Int J Eat Disorder 2020, 53:1751-1756.

6. Araujo IE de, Schatzker M, Small DM: Rethinking Food Reward. Annu Rev Psychol 2017, 71:1-26.

7. Berthoud H-R, Morrison CD, Ackroff K, Sclafani A: Learning of food preferences: mechanisms and implications for obesity \& metabolic diseases. Int J Obesity 2021, 45:21562168.

8. Seabrook LT, Borgland SL: The orbitofrontal cortex, food intake and obesity. J Psychiatry Neurosci 2020, 45:304-312.

9. Clithero JA, Rangel A: Informatic parcellation of the network involved in the computation of subjective value. Soc Cogn Affect Neur 2014, 9:1289-1302.

10. Becker GM, Degroot MH, Marschak J: Measuring utility by a single-response sequential method. Behav Sci 1964, 9:226-232.

11. Gross J, Woelbert E, Zimmermann J, Okamoto-Barth S, Riedl A, Goebel R: Value Signals in the Prefrontal Cortex Predict Individual Preferences across Reward Categories. $J$ Neurosci 2014, 34:7580-7586.

12. McNamee D, Rangel A, O'Doherty JP: Category-dependent and category-independent goal-value codes in human ventromedial prefrontal cortex. Nat Neurosci 2013, 16:479.

13. Chikazoe J, Lee DH, Kriegeskorte N, Anderson AK: Population coding of affect across stimuli, modalities and individuals. Nat Neurosci 2014, 17:1114-1122. 
14. O'Doherty JP, Rutishauser U, ligaya K: The hierarchical construction of value. Curr Opin Behav Sci 2021, 41:71-77.

15. Magrabi A, Ludwig VU, Stoppel CM, Paschke LM, Wisniewski D, Heekeren HR, Walter $\mathrm{H}$ : Dynamic Computation of Value Signals via a Common Neural Network in Multi-Attribute Decision-Making. Soc Cogn Affect Neur 2021, doi:10.1093/scan/nsab125.

16. Hare TA, Malmaud J, Rangel A: Focusing Attention on the Health Aspects of Foods Changes Value Signals in vmPFC and Improves Dietary Choice. J Neurosci 2011, 31:11077-11087.

17. Hare TA, Camerer CF, Rangel A: Self-Control in Decision-Making Involves Modulation of the vmPFC Valuation System. Science 2009, 324:646-648.

18. Tusche A, Hutcherson CA: Cognitive regulation alters social and dietary choice by changing attribute representations in domain-general and domain-specific brain circuits. Elife 2018, 7:e31185.

*This study characterized how information about tastiness, healthiness and the integrated value of the food are represented in the brain and how the representations are modulated by cognitive regulation.

19. Londerée AM, Wagner DD: The orbitofrontal cortex spontaneously encodes food health and contains more distinct representations for foods highest in tastiness. Soc Cogn Affect Neur 2020, 16:816-826.

20. Maier SU, Beharelle AR, Polanía R, Ruff CC, Hare TA: Dissociable mechanisms govern when and how strongly reward attributes affect decisions. Nat Hum Behav 2020, 4:949-963.

21. Lloyd EC, Shehzad Z, Schebendach J, Bakkour A, Xue AM, Assaf NF, Jilani R, Walsh BT, Steinglass J, Foerde K: Food Folio by Columbia Center for Eating Disorders: A Freely Available Food Image Database. Front Psychol 2020, 11:585044.

22. Sullivan N, Hutcherson C, Harris A, Rangel A: Dietary Self-Control Is Related to the Speed With Which Attributes of Healthfulness and Tastiness Are Processed. Psychol Sci 2014, 26:122-134.

23. Sullivan NJ, Huettel SA: Healthful choices depend on the latency and rate of information accumulation. Nat Hum Behav 2021, doi:10.1038/s41562-021-01154-0.

*This study showed that human food choice can be captured by a multi-attribute timedependent drift-diffusion model that considers differential influences of healthiness and tastiness on the decision process at a distinct time. 
24. Merchant JS, Cosme D, Giuliani NR, Dirks B, Berkman ET: Neural Substrates of Food Valuation and Its Relationship With BMI and Healthy Eating in Higher BMI Individuals. Front Behav Neurosci 2020, 14:578676.

25. Xue AM, Foerde K, Walsh BT, Steinglass JE, Shohamy D, Bakkour A: Neural Representations of Food-Related Attributes in the Human Orbitofrontal Cortex during Choice Deliberation in Anorexia Nervosa. J Neurosci Official J Soc Neurosci 2021, 42:109-120. *This study characterized how information about tastiness, healthiness and the integrated value of the food are represented in patients with anorexia nervosa.

26. Dalton B, Foerde K, Bartholdy S, McClelland J, Kekic M, Grycuk L, Campbell IC, Schmidt U, Steinglass JE: The effect of repetitive transcranial magnetic stimulation on food choice-related self-control in patients with severe, enduring anorexia nervosa. Int J Eat Disorder 2020, 53:1326-1336.

27. Suzuki S, Cross L, O'Doherty JP: Elucidating the underlying components of food valuation in the human orbitofrontal cortex. Nat Neurosci 2017, 20:1780-1786.

*This study demonstrated a linear combination of the nutritive attributes is predictive of the subjective value of the food, and further suggests a functional dissociation between the lateral and medial OFC in the food valuation.

28. Huang F-Y, Sutcliffe MPF, Grabenhorst F: Preferences for nutrients and sensory food qualities identify biological sources of economic values in monkeys. Proc National Acad Sci 2021, 118:e2101954118.

29. Tang DW, Fellows LK, Dagher A: Behavioral and Neural Valuation of Foods Is Driven by Implicit Knowledge of Caloric Content. Psychol Sci 2014, 25:2168-2176.

30. Motoki K, Saito T, Suzuki S, Sugiura M: Evaluation of energy density and macronutrients after extremely brief time exposure. Appetite 2021, 162:105143.

31. Öngür D, Price JL: The Organization of Networks within the Orbital and Medial Prefrontal Cortex of Rats, Monkeys and Humans. Cereb Cortex 2000, 10:206-219.

32. Howard JD, Kahnt T: To Be Specific: The Role of Orbitofrontal Cortex in Signaling Reward Identity. Behav Neurosci 2021, 135:210-217.

33. DiFeliceantonio AG, Coppin G, Rigoux L, Thanarajah SE, Dagher A, Tittgemeyer M, Small DM: Supra-Additive Effects of Combining Fat and Carbohydrate on Food Reward. Cell Metab 2018, 28:33-44.e3. 
34. Pham TQ, Yoshimoto T, Niwa H, Takahashi HK, Uchiyama R, Matsui T, Anderson AK, Sadato N, Chikazoe J: Vision-to-value transformations in artificial neural networks and human brain. Biorxiv 2021, doi:10.1101/2021.03.18.435929.

35. ligaya K, Yi S, Wahle IA, Tanwisuth K, O'Doherty JP: Aesthetic preference for art can be predicted from a mixture of low- and high-level visual features. Nat Hum Behav 2021, 5:743755.

36. Krizhevsky A, Sutskever I, Hinton GE: ImageNet classification with deep convolutional neural networks. Commun Acm 2017, 60:84-90.

37. Yamins DLK, Hong H, Cadieu CF, Solomon EA, Seibert D, DiCarlo JJ: Performanceoptimized hierarchical models predict neural responses in higher visual cortex. Proc National Acad Sci 2014, 111:8619-8624.

38. ligaya K, Yi S, Wahle IA, Tanwisuth K, O'Doherty JP: Aesthetic preference for art emerges from a weighted integration over hierarchically structured visual features in the brain. Biorxiv 2020, doi:10.1101/2020.02.09.940353.

*This study suggested a hierarchical architecture for the valuation of visual arts in the brain by leveraging a deep neural network model combined with behavioural and neuroimaging experiments.

39. Izuma K, Matsumoto M, Murayama K, Samejima K, Sadato N, Matsumoto K: Neural correlates of cognitive dissonance and choice-induced preference change. Proc National Acad Sci 2010, 107:22014-22019.

40. Wimmer GE, Shohamy D: Preference by Association: How Memory Mechanisms in the Hippocampus Bias Decisions. Science 2012, 338:270-273.

41. Nook EC, Zaki J: Social Norms Shift Behavioral and Neural Responses to Foods. J Cognitive Neurosci 2015, 27:1412-1426.

42. Barron HC, Dolan RJ, Behrens TEJ: Online evaluation of novel choices by simultaneous representation of multiple memories. Nat Neurosci 2013, 16:1492-1498.

43. Araujo IE de, Rolls ET: Representation in the Human Brain of Food Texture and Oral Fat. J Neurosci 2004, 24:3086-3093.

44. Grabenhorst F, Rolls ET, Parris BA, d'Souza AA: How the Brain Represents the Reward Value of Fat in the Mouth. Cereb Cortex 2010, 20:1082-1091.

45. Grabenhorst F, Rolls ET: The representation of oral fat texture in the human somatosensory cortex. Hum Brain Mapp 2014, 35:2521-2530. 
46. Small DM, Gregory MD, Mak YE, Gitelman D, Mesulam MM, Parrish T: Dissociation of Neural Representation of Intensity and Affective Valuation in Human Gustation. Neuron 2003, 39:701-711.

47. Chikazoe J, Lee DH, Kriegeskorte N, Anderson AK: Distinct representations of basic taste qualities in human gustatory cortex. Nat Commun 2019, 10:1048.

48. Avery JA, Liu AG, Ingeholm JE, Riddell CD, Gotts SJ, Martin A: Taste Quality Representation in the Human Brain. J Neurosci 2019, 40:1042-1052.

49. Avery JA, Liu AG, Ingeholm JE, Gotts SJ, Martin A: Viewing images of foods evokes taste quality-specific activity in gustatory insular cortex. Proc National Acad Sci 2021, 118:e2010932118.

50. Nakamura Y, Imafuku M, Nakatani H, Nishida A, Koike S: Difference in neural reactivity to taste stimuli and visual food stimuli in neural circuits of ingestive behavior. Brain Imaging Behav 2020, 14:1395-1405.

51. Schultz W, Dayan P, Montague PR: A Neural Substrate of Prediction and Reward. Science 1997, 275:1593-1599.

52. Sclafani A: Oral and postoral determinants of food reward. Physiol Behav 2004, 81:773779.

53. Blechert J, Lender A, Polk S, Busch NA, Ohla K: Food-Pics_Extended_An Image Database for Experimental Research on Eating and Appetite: Additional Images, Normative Ratings and an Updated Review. Front Psychol 2019, 10:307. 


\section{Figures}

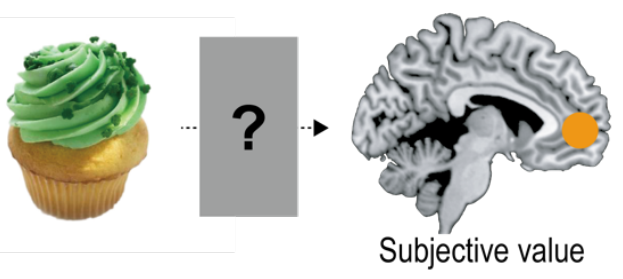

Figure 1: The ventromedial prefrontal cortex (vmPFC: orange area) have been shown to represent subjective value signals; however, the process by which the value signals are constructed remains elusive. The food image is extracted from Foodpics [53].

a

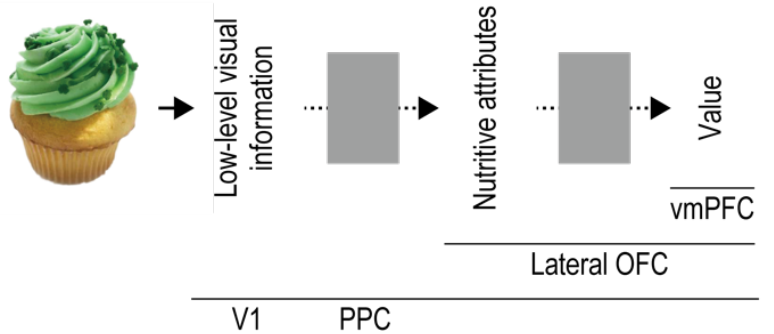

b

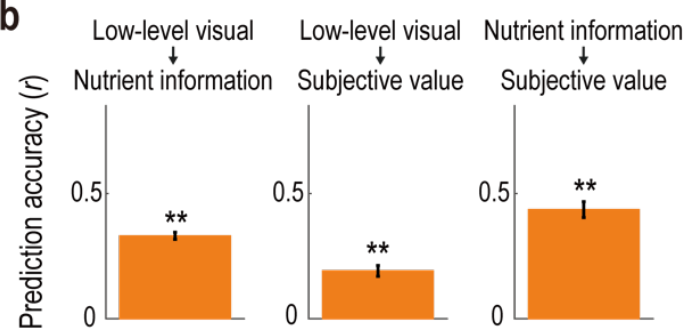

Figure 2: (a) Hierarchical architecture for food valuation. V1, primary visual cortex; PPC, posterior parietal cortex; Lateral OFC, lateral orbitofrontal cortex; and vmPFC, ventromedial prefrontal cortex including medial OFC. (b) Results of the additional behavioural analysis on the published data [27]. The accuracy is evaluated by cross-validated (leave-one-image-out) correlations between the actual and the predicted values. Left, prediction accuracy of the nutrient information from the low-level visual information (colour intensities, hue, saturation, brightness, and local contrast). The averaged accuracy over the four nutrients (fat, carbohydrate, protein, vitamin) is shown. ${ }^{* *} P<0.001$ (permutation test). Centre, prediction accuracy of the subjective value from the low-level visual information. Right, prediction accuracy of the subjective value from the nutrient information. 